\title{
Mucormycosis in a pediatric population: a review of 20 cases from southern Turkey
}

\author{
Derya Alabaz ${ }^{1 \oplus,}$ Gülsüm Yılmaz ${ }^{1 \oplus}$, Aysun Uğuz ${ }^{2 \oplus}$, Süleyman Özdemir ${ }^{3 \oplus}$, \\ İlgen Şaşmaz ${ }^{4 \oplus}$, İbrahim Bayram ${ }^{4}$ \\ Departments of ${ }^{1}$ Pediatric Infectious Disease, ${ }^{2}$ Pathology, ${ }^{3}$ Otolaryngology-Head and Neck Surgery, and ${ }^{4}$ Pediatric Hematology and \\ Oncology, Çukurova University Faculty of Medicine, Adana, Turkey.
}

\begin{abstract}
Background. Mucormycosis is a fatal invasive fungal infection seen most often in patients with compromised defense mechanisms. The aim of this article was to review the data of pediatric mucor in the South of Turkey.

Methods. Twenty pediatric cases with biopsy proven mucormycosis were reported, between January 2007 through January 2017. Data were extracted from the medical charts of patients retrospectively.

Results. Underlying conditions were hematological malignancy (75\%), in whom 93\% had acute leukemia, aplastic anemia $(15 \%)$, diabetes mellitus $(5 \%)$ and other malignancies $(5 \%)$. The main sites of infection were sinus $(85 \%)$; alone $(29.4 \%)$ or with cerebral $(17.6 \%)$, and orbital involvement $(17.6 \%)$. Pulmonary involvement was reported in 11 patients (55\%), two of them had the alone form and nine cases were associated with nasal sinus involvement. Disseminated mucormycosis was documented in $45 \%$. Fever and pain/swelling of organs were the most commonly encountered signs and symptoms. Treatment compromised of am-photericin B monotherapy in five patients. All patients except one received liposomal formu-lations (LAmB). A combination of surgery and antifungal therapy was performed in $75 \%$. Crude survival was 55\%; among 15 cases treated with a combination of surgery and antifun-gal therapy, survival rate was $8 / 15(53 \%)$. The overall mortality rate was high in patients diagnosed with disseminated infection (100\%).
\end{abstract}

Conclusions. Mucormycosis in pediatric cases requires a high index of suspicion and urgent evaluation of clinical samples. Surgical debridement should be considered when feasible. Initial medical therapy should include an amphotericin preparation with or step-down to posaconazole.

Key words: mucormycosis, zygomycosis, children, immunosuppressive, amphotericin B.

Mucormycosis (zygomycosis) is the third emerging important invasive fungal infection dur-ing the past decade, it is associated with a worse outcome when compared to other invasive fungal infections such as candidiasis or aspergillosis. ${ }^{1-4}$ The increase in the incidence may be attributed to a better outcome in the survival of immunocompromised patients. ${ }^{5}$ Depending on the underlying condition, such as the withdrawal or reduction of corticosteroids, impair-ment of neutropenia,

\footnotetext{
$\triangle$ Derya Alabaz

deryaalabaz@yahoo.com
}

Received 14th September 2019, revised 17th April 2020, accepted 5th June 2020. hematological malignancies, hematopoietic stem cell transplantation (HSCT), adequate control of glycemia in cases of diabetes, and the portal of entry, they can cause rhinocerebral, pulmonary, cutaneous, gastrointestinal or even disseminated infection. ${ }^{6,7}$ The rapid initiation of antifungal therapy is the cornerstone due to the highly difficult treat-ment of this destructive infection.

We report retrospective results in the present study, where we reviewed clinical characteristics, risk factors, treatment and outcome of pediatric mucormycosis, diagnosed by histopathology at our center from 2007 until 2017, a University Hospital in Southern Turkey. 


\section{Material and Methods}

The study was conducted in a tertiary medical college hospital (Çukurova University Faculty of Medicine). Our patients were managed by a multidisciplinary healthcare team comprised of oncologists, infectious disease specialists, surgeons, pathologists, radiologists and inten-sivists. The study was approved by the Institutional Ethics Committee (Approval number: 04-09-2019/91).

All pediatric patients (aged 0-18) that were diagnosed histologically as mucormycosis between January 2007 through January 2017, were included in the study. All cases were proven as mucormycosis based on a histopathologic examination of a needle aspiration and/or a bi-opsy specimen which revealed hyphae and evidence of associated tissue damage from the nasal cavity and/or paranasal sinuses and/or palate, lung or dermis.

Histopathologic diagnosis: Mucor infections are one of the correctly detectable fungi by biopsy and they are determined as angioinvasive, broad, without septas and 90 degrees branching hyphae in tissue sections. Although routine hematoxylene-eosin tissue sections are generally sufficient some histochemical stains like PAS and GMS (Gomori's methanamine silver) can be used, its particularly predominant appearance is necrosis. Sometimes fungal hyphaes can be few, or degraded or folded in tissue sections.

All patients underwent computed tomography (CT) or magnetic resonance imaging (MRI) scans of paranasal sinuses, orbita, craniocerebrum and lungs.

The sites of infection were classified according to those utilized in the study of Roden et al. ${ }^{4}$ Accordingly, sinusitis was defined as an infection involving the paranasal sinuses; those with disease in paranasal sinuses and orbital infiltration were defined as sino-orbital infec-tion; those with disease in the paranasal sinuses and the brain as rhinocerebral infection; those with disease in the paranasal sinuses and lungs were defined as having sinopulmonary infection; pulmonary infection was defined as infection confined to lung tissue and as deep extension when invading adjacent tissues, and dissemination was defined as two or more noncontiguous locations of Mucorales infection.

The demographic characteristics, type of underlying conditions, risk factors, the site of in-fection, clinical signs and symptoms of infection, radiological findings, treatments, and out-come were extracted from the medical charts of patients.

\section{Results}

The hospital pathology records identified 24 individual cases of mucormycosis in pediatric patients during 10 years. Of these, four cases were excluded from the database because they did not meet the stringent predefined inclusion criteria or the patient file could not be reached. During the study, 20 patients with proven mucormycosis (14 male, 6 female) were recorded. Demographic characteristics and clinical features are summarized in Tables I and II. The age at diagnosis ranged from 2 to 16 years (average age was 9 years) and 7 patients were aged $\leq 5$ years. Two patients out of 20 were foreign (Syrian) nationals.

The patients' underlying conditions are listed in Table II. Fifteen patients (75\%) with hematological malignancy composed the largest group, in whom 93\% had acute leukemia (one underwent HSCT). Three patients had aplastic anemia ( 2 were Fanconia anemia and one underwent HSCT). Eighteen patients were neutropenic and 8 patients were known as being on antifungal prophylaxis (5 patients with fluconazole and 3 patients with itroconazole) while 7 patients were receiving co-trimaxazole. Two patients had chronic renal failure and one patient had metabolic acidosis.

Nasal involvement was identified in a majority of the cases $(17 / 20 ; 85 \%)$ patients, alone in $5 / 17$, with cerebral involvement in $3 / 17$, and with orbital involvement in 3/17 (Table III). Pulmonary involvement was reported in $11 / 20$ 


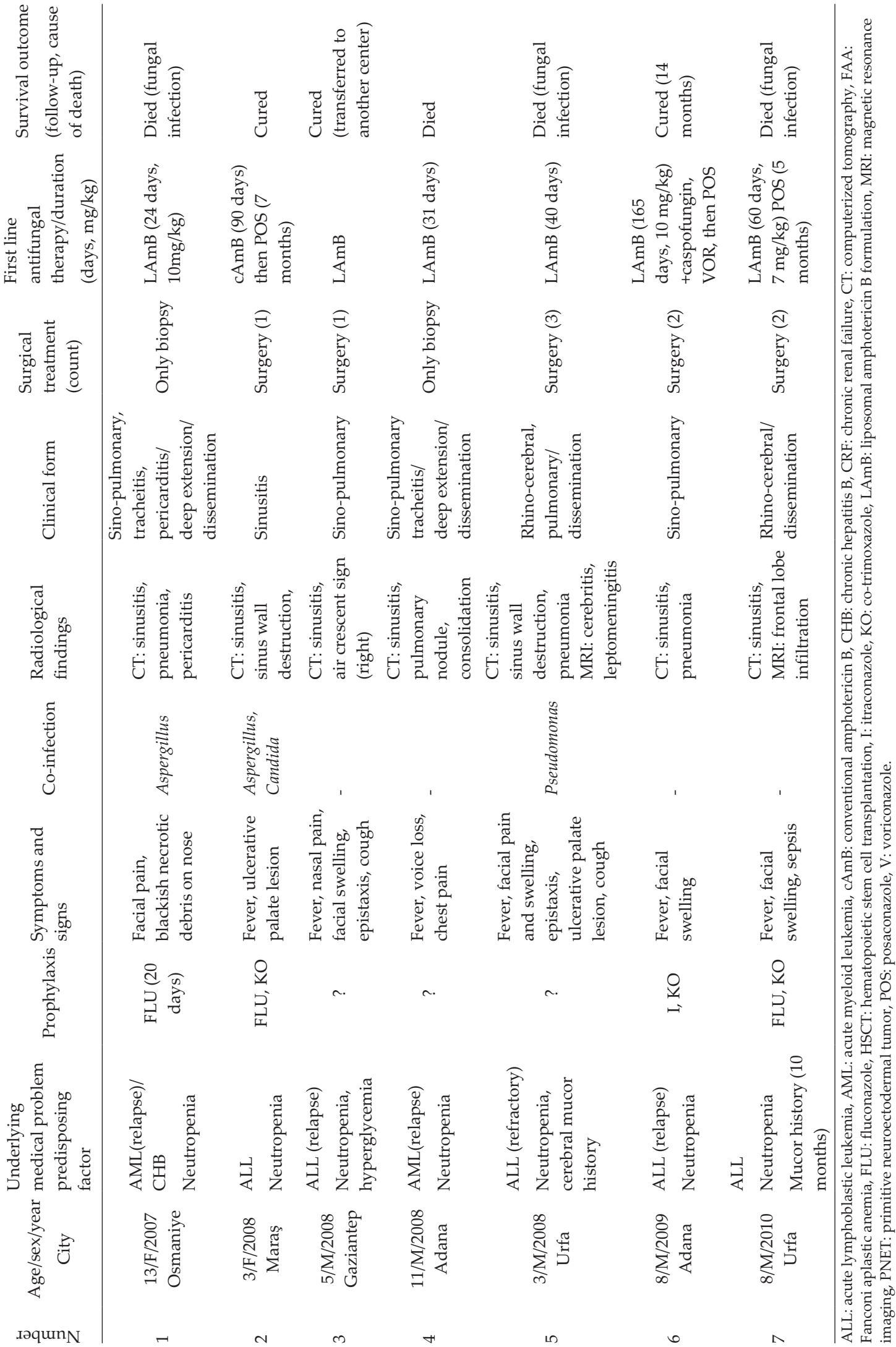




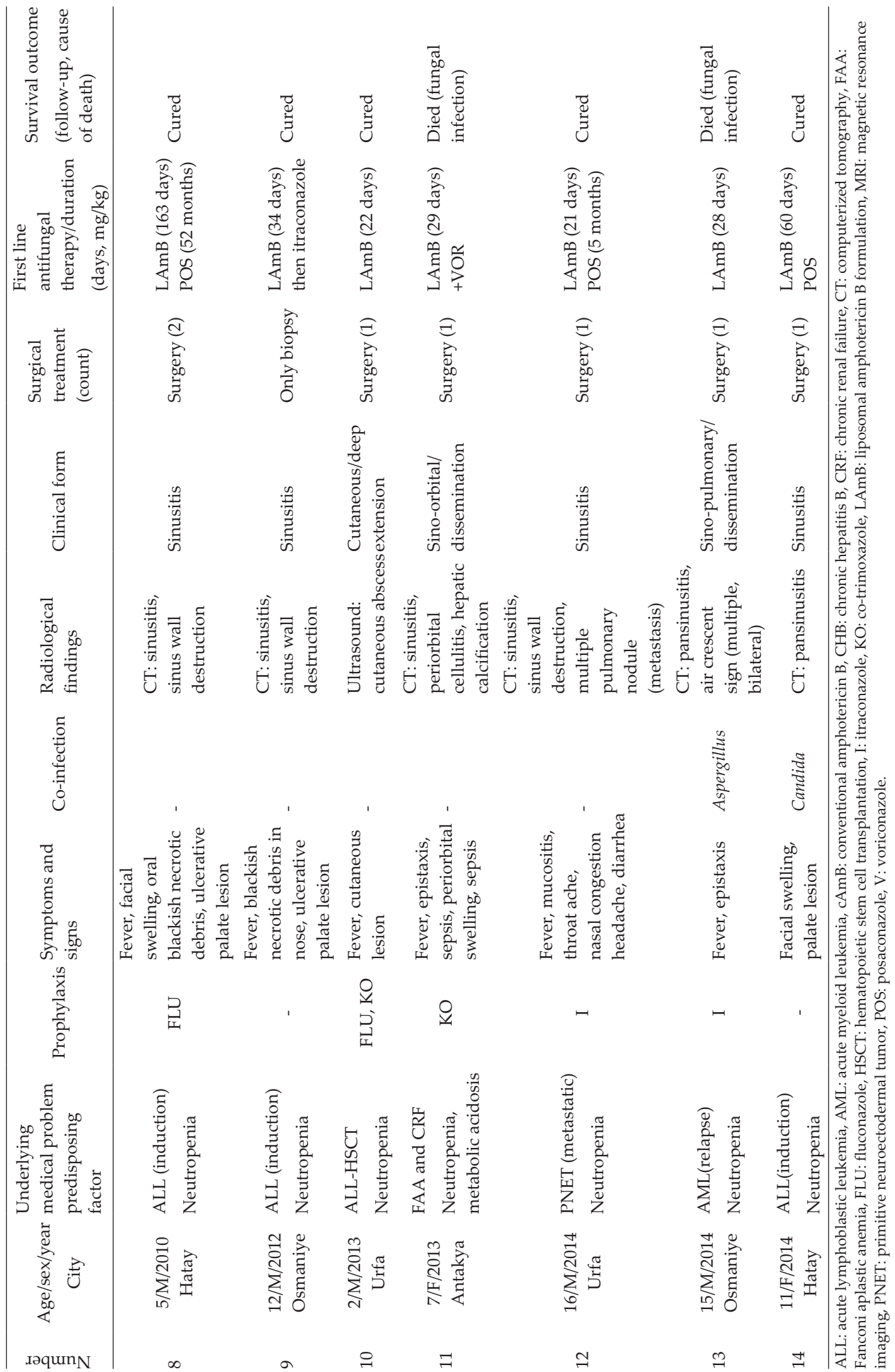




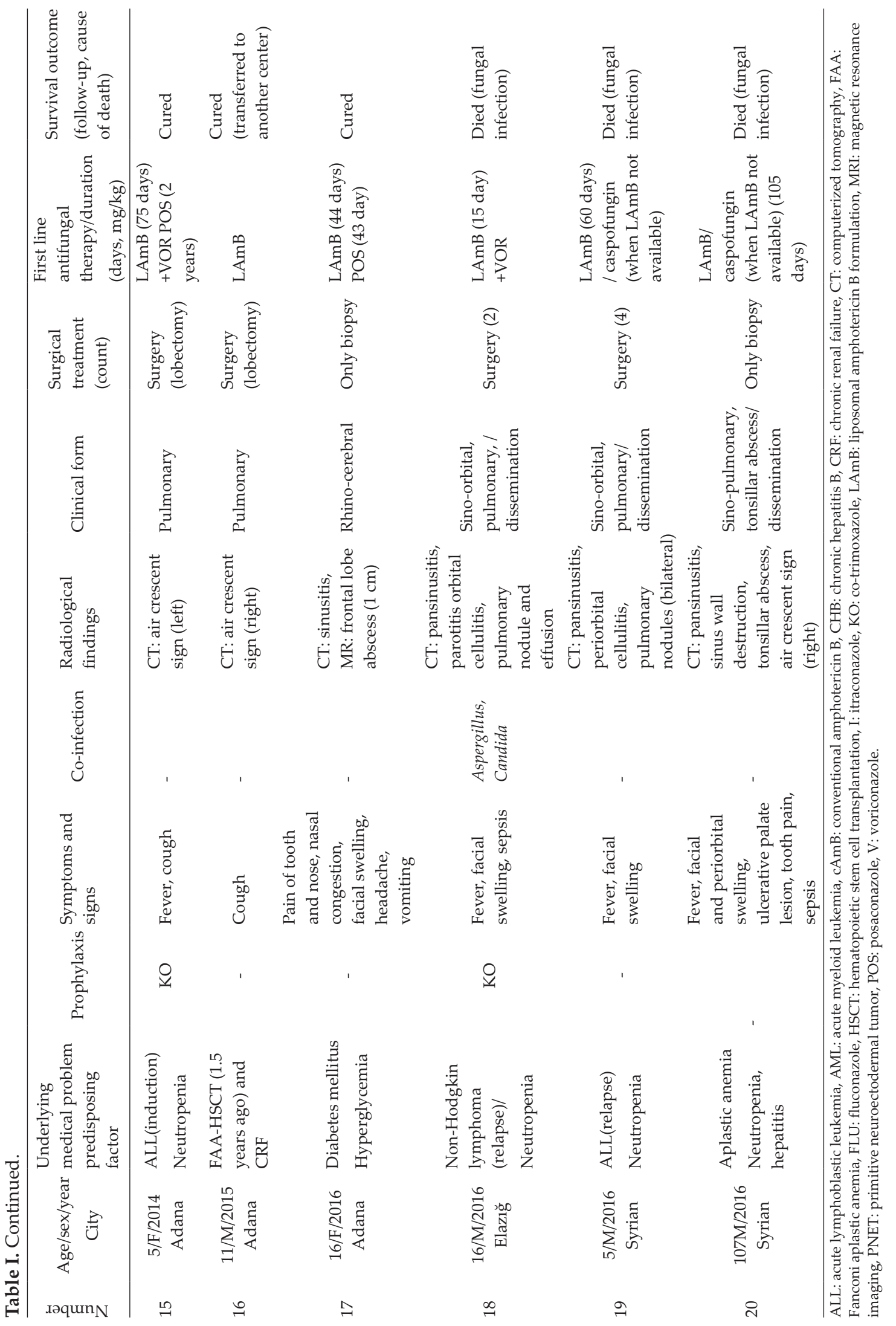


Table II. Patient demographics, predisposing factors, clinical findings and outcomes.

\begin{tabular}{|c|c|c|}
\hline Clinical features & $\mathrm{N}(\%)$ & Mortality, $\mathrm{n} / \mathrm{N}(\%)$ \\
\hline \multicolumn{3}{|l|}{ Age } \\
\hline$<5$ years & $3(15)$ & 1/9 (11) \\
\hline$\geq 5-18$ years & $17(85)$ & $8 / 9(89)$ \\
\hline Male/Female & $14 / 6$ & $7 / 14 ; 2 / 6(50 ; 33)$ \\
\hline \multicolumn{3}{|l|}{ Co-morbidity } \\
\hline Diabetes mellitus & $1(5)$ & - \\
\hline Haematological malignancy & $15(75)$ & $9 / 15(60)$ \\
\hline ALL & $11(55)$ & $3 / 11(27)$ \\
\hline AML & $3(15)$ & $3 / 3(100)$ \\
\hline Non-Hodgkin lymphoma & $1(5)$ & $1 / 1(100)$ \\
\hline \multicolumn{3}{|l|}{ Other malignancies } \\
\hline PNET & $1(5)$ & - \\
\hline \multicolumn{3}{|l|}{ Haematological disease } \\
\hline Aplastic anemia & $3(15)$ & $2 / 3(67)$ \\
\hline \multicolumn{3}{|l|}{ Predisposing factor } \\
\hline Neutropenia & $18(90)$ & $9 / 18(50)$ \\
\hline Cancer chemotherapy & $16(80)$ & $7 / 16(44)$ \\
\hline Co-infection with Aspergillus & $5(25)$ & $2 / 5(40)$ \\
\hline Co-infection with Candida & $3(15)$ & $2 / 3(67)$ \\
\hline \multicolumn{3}{|l|}{ Antifungal prophylaxis medication } \\
\hline Fluconazole & $5(25)$ & $2 / 5(40)$ \\
\hline Itraconazole & $3(15)$ & $1 / 3(33)$ \\
\hline Co-Trimoxazole & $7(35)$ & \\
\hline \multicolumn{3}{|l|}{ Signs and symptoms } \\
\hline Fever & $16(80)$ & \\
\hline Swelling of face/periorbital & $12(72)$ & \\
\hline Blackish necrotic debris of nose/oral & $8(40)$ & \\
\hline Ulcerative palate lession & $6(30)$ & \\
\hline Facial/nasal/tooth/throat pain and tooth infection & $6(30)$ & \\
\hline Bloody nasal discharge & $4(20)$ & \\
\hline Cough & $4(20)$ & \\
\hline Sepsis & $4(20)$ & \\
\hline Headache & $2(10)$ & \\
\hline Nasal congestion & $2(10)$ & \\
\hline Voice loss & $1(5)$ & \\
\hline Chest pain & $1(5)$ & \\
\hline Surgery & $15(75)$ & \\
\hline \multicolumn{3}{|l|}{ Outcomes } \\
\hline Mortality & $9(45)$ & \\
\hline
\end{tabular}

(55\%) patients, 2 of them had alone form and 9 associated with nasal sinus involvement. Disseminated mucormycosis was documented in $9 / 20(45 \%)$ patients. There were unusual presentations, including 2 patients with parotitis and hepatitis. 


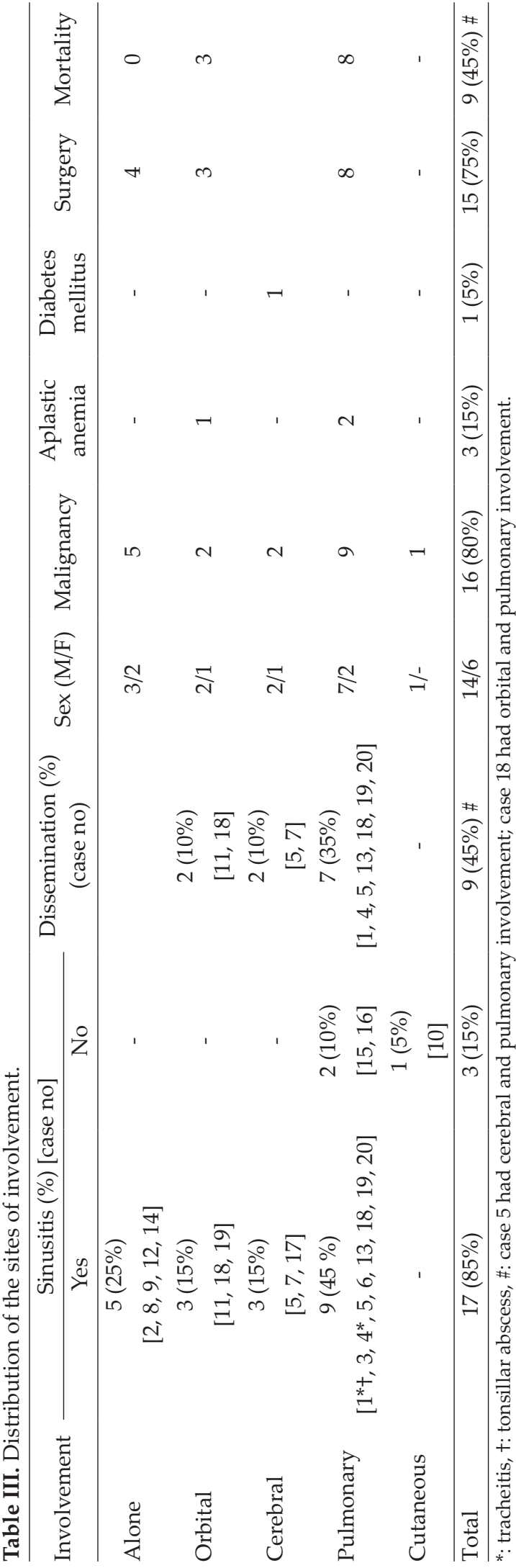

Generally, fever and pain/swelling of organs were the most commonly encountered signs and symptoms (Table II). None of the patients were culture positive. In cases accompanied by Aspergillus, 3 of the 4 cases showed death and worsened prognosis.

Treatment regimens prescribed for patients diagnosed with mucormycosis are shown in Table II. All patients were treated with a form of antifungal therapy, resulting in a survival of $55 \%$. All patients except one (whom had conventional amphotericin B, cAmB) received lip-osomal formulations (LAmB). Fifteen of these cases were treated with a combination of sur-gery and antifungal therapy, resulting in a survival rate of $8 / 15$ (53\%) (Table II). Posaconazole has been used successfuly for step-down therapy in seven patients. The overall mortality rate of patients identified in this study was $45 \%$, with a high mortality rate occur-ring in patients diagnosed with disseminated infection (100\%).

\section{Discussion}

Mucormycosis is a life-threatening fungal infection characterized by a highly aggressive (angiotropic) progression that occurs mostly in immunocompromised patients. ${ }^{8}$ Data concerning patient sex, age at onset of disease, symptoms and signs, radiological findings, treatment modalities, and outcome were analyzed retrospectively, for a 10-year period. To our knowledge, there have been a limited number of reports from Turkey in the pediatric population diagnosed with mucormycosis, therefore, our report is a large case series reported from our country.

In several studies, mucormycosis was reported primarily in males (65\%). In case reports from Turkey, most of the patients were male (52\%). ${ }^{9}$ Although Pana et al. ${ }^{1}$, in 63 children diagnosed with mucormycosis, reported the rate of girls as higher, in our study, a significant proportion of the patients were male. In our series, the majority of children (85\%) were older than 5 years, with a mortality of $78 \%$. 
For older infants and children, Francis et al. $^{10}$ has recently reported that hematologic malignancy is a significant risk factor, as is being a hematopoietic stem cell transplant or solid organ transplant recipient, these were followed by diabetes. Kömür et al. ${ }^{11}$ conducted a retrospective study evaluating 51 cases of mucormycosis in our city in adult patients and reported that malignancy was the most common co-morbidity affecting 59\% of the subjects, followed by diabetes. In our series of 20 pediatric mucormycosis cases, the most prominent identified underlying conditions were hematological malignancies, especially acute leukemia (70\%), and this was followed by aplastic anemia (15\%). In patients with prolonged neutropenia, aggressive evaluation should be performed in case of fever. ${ }^{12}$ The majority of patients in our series had neutropenia $(90 \%)$. Fever was observed in almost every neutropenic case. Although, we have a well working transplantation unit, only two $(10 \%)$ of our pediatric mucor patients were transplant patients; the distribution of infection is different from the literature. In our experience, the mucor infection occurred in four patients in the early stage of chemotherapy, during induction, and that contrasts with the data in the literature of pediatric mucor cases in which this was reported to occur later on during chemotherapy. ${ }^{13}$ Seven of the ALL cases were either relapsed or refractory and clinically unwell. In our study, in only one of 20 cases, the patient had uncontrolled diabetes mellitus.

Seasonal variation in atmospheric concentration of fungal spores has been documented for some molds in several geogaphical locations. ${ }^{14,15}$ As reported in India and in Middle East countries such as Iran, events are reproduced in August and in tropical and subtropical sea-sons when spores are most intense in the air. ${ }^{16}$ It is necessary to evaluate the contribution of this climate zone and the seasonal weather conditions we have in our city. The fact that our region is moist might be an effective risk factor for disease development.
The authors' prior and most recent experience with hematological malignancy patients suggests that mucormycosis is most likely in cases when the patient has been receiving As-pergillus-active antifungal (especially voriconazole) prophylaxis. ${ }^{17-19}$ Although in our series, $8 / 20$ patients who had received antifungal prophylaxis with fluconazole or itraconazole, did not have Mucorales activity.

For Mucorales the portals of entry in the human body are the respiratory tract through inhalation of fungal spores. In most series ${ }^{20-25}$, such as the studies of Chakrabarti et $\mathrm{al}^{3}{ }^{3}$ and Roden et $\mathrm{al}^{4}{ }^{4}$, rhino-orbito-cerebral mucormycosis was reported as the most frequent clinical mani-festation and this can quickly progress to disseminated form with disastrous consequences if not diagnosed and treated early. This is consistent with the global trend, the most commonly identified condition was sinus involvement (75\%) alone in 5/20 and with concomitant in-volvement in $12 / 20$ in our study, followed by the pulmonary form, have been found to be the most prevalent in our study. Pulmonary mucormycosis has been reported at a frequency be-tween $44-64 \%$ in children. ${ }^{26-29}$ Although rhinoorbitocerebral sinus disease is mostly common in diabetic patients, pulmonary disease predominates in pediatric patients with malignancy and hematopoietic cell transplantations $(75 \%) .{ }^{1}$ We detected that eleven cases (55\%) had pulmonary involvement (9/11 had added involvement of other forms). Seven $(7 / 11,78 \%$ ) with pulmonary involvement occurred as the disseminated form. From a recent review of Francis et al. ${ }^{10}$ the dissemination form of the disease occurs in 32\% to $38 \%$ of pediatric cas-es, which was $45 \%$ in our cases, higher than the literature. The patients with a single in-volvement form had a better outcome than those with dissemination. ${ }^{30}$

Histopathologic examination of clinical specimens and culture are recommended for the diagnosis of mucormycosis. Although tissues frequently are not available for biopsy, because of thrombocytopenia or hemodynamic instability, definitive diagnosis is made most frequently 
on the basis of direct microscopic examination. However, tissue identification is a very important diagnostic tool, since it distinguishes the presence of the fungus as a pathogen in the specimen from a culture contaminant. Direct microscopy of clinical specimens allows a rapid presumptive diagnosis and differentiation of mucormycosis from aspergillosis and other hyalohyphomycoses and phaeohyphamycosesand is strongly recommended for treatment decisions. In recent registries of mucormycosis, histopathology led to the diagnosis in $63 \%$ and $66 \%$ of cases. ${ }^{31,32}$ The diagnosis of 75 cases from an Indian tertiary-care hospital was based on histopathology. ${ }^{33}$

Culture of a clinically relevant isolate enables identification and susceptibility testing of the pathogen. Culture is poorly sensitive because Mucorales hyphae are friable in nature, hence may be damaged easily during sample collection (avoidance of excessive tissue homogenization is recommended before culturing). Additionally, some species fall to sporulate in standard media, precluding a timely and easy morphological identification. Better recovery is seen if slices of minimally manipulated tissue are placed onto the culture medium or baited with bread to promote mycelial growth. ${ }^{34,35}$ As a result, approximately only one-third of all histopatologically proven specimens result in a positive fungal culture. ${ }^{36,37}$ Countless reports of negative culture results are scattered throughout the literature.

Imaging techniques are helpful; although they are non-specific and do not correlate well with surgical and pathological findings. According to the revised version of EORTC/MSG pub-lished in $2008^{38}$, all of our patient's diagnosis were documented with proven mucormycosis by histopathology and none of them were culture positive. As our opinion, in our center neg-ative culture may be explained by various factors, such as aggressive processing of the spec-imen and inappropriate storage of samples before plating. Although the limitation in our study was biased by the selection of cases that were only proven by biopsy, the diagnosis of our patients was confirmed by histopathological and radiological examination in addition to clinical findings. Despite the limitations to the study, we retrospectively gave a good esti-mate for the burden of mucor infections in pediatric cases in Turkey, highlighting the index of clinical suspicion and the important role played by histology especially from suspected cases in the diagnosis that will be able to guide towards early surgery.

The mucormycosis treatment with antifungal medicine is an important factor affecting the outcome. According to the literature, the mainstay of therapy for treating mucor remains as amphotericin $B$, primarily in its liposomal formulation. ${ }^{39}$ Furthermore, delayed antifungal therapy would increase mortality of mucormycosisamong patients withneutropenia. In our series, the first line treatment was cAmB or LAmB in all patients, at different daily doses up to $10 \mathrm{mg} / \mathrm{kg}$. In our experience, higher doses of LAmB were well tolerated. For combination therapy, Pagano et al..$^{41}$ reported the beneficial effect of posaconazole in addition to LAmB in hematological patients failing to response to LAmB monotherapy. ${ }^{40-43}$ Though posaconazole was not available to give in the first years in our study. In recent years, 7 patients were given parenteral $\mathrm{AmB}$ followed by descalation to oral posaconazole. The optimal total duration of antifungal drug administration required for mucormycosis is controversial and varies depending on the extent of the disease. ${ }^{44}$ In children successfully treated in our series, we discontinued antifungal therapy only when clinical resolution was evident and adequate im-mune recovery had occurred.

Because of the risk of rapid progression to dissemination of pediatric mucormycosis cases, when feasible, surgery should be considered as a treatment choice. Surgery and antifungal combination therapy are mostly the mainstays of management of invasive mucormyco-sis., ${ }^{4,74}$ Children who received combined therapy had a mortality rate of $18.5 \%$ compared 
with $60 \%$ for those who received antifungal therapy alone. ${ }^{30}$ The majority of the patients suffered from serious underlying conditions (thrombocytopenia, pulmonary infection) limiting the possibility for surgery. Surgery was just performed on $15 / 20$ patients in this study. Of these cases, there was a $60 \%$ response rate.

Despite aggressive surgical intervention and intensive antifungal treatment, mucormycosis is associated with a greater mortality rate (47$56 \%$ ). It rises to range from $50-100 \%$ depending on the disease form, which is in agreement with our findings in the current study. $5,32,45,46$ Our survival rate was 55\%. Despite the fact that most of the disseminated cases received a combined treatment, the high mortality rate shows that this treatment is not sufficient.

Empirical treatment for mucormycosis is emergent if there are suspicions. The cases in our study were definitively diagnosed cases. In fact it is certain that the rate is higher than it is in this series, because we think most cases have not been appropriately diagnosed. Although surgery was performed in most of the cases, the mortality rate was high due to the severity of the disease. Perhaps an earlier intervention should be made. Unfortunately, we did not have a chance for postmortem examination to prove this claim.

In conclusion, a steady increase in the reports of mucormycosis during the last decades may be due to increased awareness of a fungal infection in risk groups, and early diagnosis and treatment of these invasive fungal infections can improve the outcomes of children. The recommended management for overall survival of invasive mucormycosis has been surgical debridement combined antifungal therapy and restoration of the underlying immune status should be considered a key factor for a better outcome of the disease. As can be seen from these, good management of risk factors, especially neutropenia and hyperglycemia, prevents this disease from occurring.

\section{REFERENCES}

1. Pana ZD, Roilides E, Warris A, Groll AH, Zaoutis T. Epidemiology of invasive fungal disease in children. J Pediatr Infect Dis Soc 2017; 6(Suppl 1): S3-S11.

2. Petrikkos G, Skiada A, Lortholary O, Roilides E, Walsh TJ, Kontoyiannis DP. Epidemiology and clinical manifestations of mucormycosis. Clin Infect Dis 2012; 54(Suppl 1): S23-S34.

3. Chakrabarti A, Singh R. The emerging epidemiology of mould infections in developing countries. Curr Opin Infect Dis 2011; 24: 521-526.

4. Roden MM, Zaoutis TE, Buchanan WL, et al. Epidemiology and outcome of zygomycosis: a review of 929 reported cases. Clin Infect Dis 2005; 41: 634-653.

5. Skiada A, Lanternier F, Groll AH, et al; European Conference on Infections in Leukemia Diagnosis and treatment of mucormycosis in patients with hematological malignancies: Guidelines from the 3rd European Conference on Infections in Leukemia (ECIL 3). Haematologica 2013; 98: 492-504.

6. Petrikkos G, Drogari-Apiranthitou M. Zygomycosis in immunocompromised non-haematological patients. Mediterr J Hematol Infect Dis 2011; 3: e2011012.

7. Cornely OA, Arıkan-Akdaglı S, Dannaoui E, et al; European Society of Clinical Microbiology and Infectious Diseases Fungal Infection Study Group; European Confederation of Medical Mycology ESCMID and ECMM joint clinical guidelines for the diagnosis and managment of mucormycosis 2013. Clin Microbiol Infect 2014; 20(Suppl 3): 5-26.

8. Bitar D, Van Cauteren D, Lanternier F, et al. Increasing incidence of zygomycosis (mucormycosis), France, 1997-2006. Emerg Infect Dis 2009; 15: 1395-1401.

9. Zeka AN, Taşbakan M, Pullukçu H, Sipahi OR, Yamazhan T, Arda B. Evaluation of zygomycosis cases by pooled analysis method reported from Turkey. Mikrobiyol Bul 2013; 47: 708-816.

10. Francis JR, Villanueva P, Bryant P, Blyth CC. Mucormycosis in children; review and recommendations for management. J Pediatric Infect Dis Soc 2018; 7: 159-164.

11. Kömür S, Inal AS, Kurtaran B, et al. Mucormycosis: a 10 -year experience at a tertiary care center in Turkey. Turk J Med Sci 2016; 46: 58-62.

12. Guymer C, Khurana S, Suppiah R, Hennessey I, Cooper C. Successful treatment of disseminated mucormycosis in a neutropenic patient with T-cell acute lymphoblastic leukaemia. BMJ Case Rep 2013; 2013: bcr2013009577. 
13. Muggeo P, Calore E, Decembrino $N$, et al. Invasive mucormycosis in children with cancer: a retrospective study from the Infection Working Group of Italian Pediatric Hematology Oncology Association. Mycoses 2019; 62: 165-170.

14. Al-Ajam MR, Bizri AR, Mokhbat J, Weedon J, Lutwick L. Mucormycosis in the Eastern Mediterranean: a seasonal disease. Epidemiol Infect 2006; 134: 341346.

15. Kontoyiannis DP, Lewis RE. Invasive zygomycosis: update on pathogenesis, clinical manifestations, and management. Infect Dis Clin North Am 2006; 20: 581-607.

16. Shpitzer T, Keller N, Wolf M, et al. Seasonal variations in rhino-cerebral Mucor infection. Ann Otol Rhinol Laryngol 2005; 114: 695-698.

17. Kontoyiannis DP, Lionakis MS, Lewis RE, et al. Zygomycosis in a tertiary-care cancer center in the era of Aspergillus-active antifungal therapy: a casecontrol observational study of 27 recent cases. J Infect Dis 2005; 191: 1350-1360.

18. Siwek GT, Pfaller MA, Polgreen PM, et al. Incidence of invasive aspergillosis among allogeneic hematopoietic stem cell transplant patients receiving voriconazole prophylaxis. Diagn Microbiol Infect Dis 2006; 55: 209-212.

19. Trifilio S, Singhal S, Williams S, et al. Breakthrough fungal infections after allogeneic hematopoietic stem cell transplantation in patients on prophylactic voriconazole. Bone Marrow Transplant 2007; 40: 451-456.

20. Pagella F, De Bernardi F, Dalla Gasperina D, et al. Invasive fungal rhinosinusitis in adult patients: our experience in diagnosis and management. J Craniomaxillofac Surg 2016; 44: 512-520.

21. Palejwala SK, Zangeneh TT, Goldstein SA, Lemole GM. An aggressive multidisciplinary approach reduces mortality in rhinocerebral mucormycosis. Surg Neurol Int 2016; 7: 61.

22. Bakhshaee M, Bojdi A, Allahyari A, et al. Acute invasive fungal rhinosinusitis: our experience with 18 cases. Eur Arch Otorhinolaryngol 2016; 273: 42814287.

23. Green KK, Barham HP, Allen GC, Chan KH. Prognostic factors in the outcome of invasive fungal sinusitis in a pediatric population. Pediatr Infect Dis J 2016; 35: 384-386.

24. Jiang N, Zhao G, Yang S, et al. A retrospective analysis of eleven cases of invasive rhino-orbitocerebral mucormycosis presented with orbital apex syndrome initially. BMC Ophthalmol 2016; 16: 10.
25. Teixeira CA, Medeiros PB, Leushner P, Almeida F. Rhinocerebral mucormycosis: literature review apropos of a rare entity. BMJ Case Rep 2013; 2013: bcr 20122008552.

26. Yan $\mathrm{X}$, Zong F, Kong $\mathrm{H}$, et al. Pulmonary fungal diseases in immunocompetent hosts: a single-center retrospective analysis of 35 subjects. Mycopathologia 2016; 181: 513-521.

27. Feng J, Sun X. Characteristics of pulmonary mucormycosis and predictive risk factors for the outcome. Infection 2018; 46: 503-512.

28. Lin E, Moua T, Limper AH. Pulmonary mucormycosis: clinical features and outcomes. Infection 2017; 45: 443-448.

29. He R, Hu C, Tang Y, Yang H, Cao L, Niu R. Report of 12 cases with tracheobronchial mucormycosis and a review. Clin Respir J 2018; 12: 1651-1660.

30. Pana ZD, Seidel D, Skiada A, et al; Collaborators of Zygomyco.net and/or FungiScope ${ }^{\mathrm{TM}}$ Registries*. Invasive mucormycosis in children: an epidemiologic study in European and non-European countries based on two registries. BMC Infect Dis 2016; 16: 667.

31. Rüping MJ, Heinz WJ, Kindo AJ, et al. Forty-one recent cases of invasive zygomycosis from a global clinical registry. J Antimicrob Chemother 2010; 65: 296-302.

32. Skiada A, Pagano L, Groll A, et al; European Confederation of Medical Mycology Working Group on Zygomycosis. Zygomycosis in Europe: analysis of 230 cases accrued by the registry of the European Confederation of MedicalMycology (ECMM) Working Group on Zygomycosis between 2005 and 2007. Clin Microbiol Infect 2011; 17: 18591867.

33. Chakrabarti A, Chatterjee SS, Das A, et al. Invazive zygomycosis in India: experience in a tertiary care hospital. Postgrad Med J 2009; 85: 573-581.

34. Rinaldi MG. Zygomycosis. Infect Dis Clin North Am 1989; 3: 19-41.

35. Ribes JA, Vanover-Sams CL, Baker DJ. Zygomycetes in human disease. Clin Microbiol Rev 2000; 13: 236301.

36. Walsh TJ, Gamaletsou MN, McGinnis MR, Hayden RT, Kontoyiannis DP. Early clinical and laboratory diagnosis of invasive pulmonary, extrapulmonary and disseminated mucormycosis (zygomycosis). Clin Infect Dis 2012; 54(Suppl 1): S55-S60.

37. Lackner M, Caramalho R, Lass-Flörl C. Laboratory diagnosis of mucormycosis: current status and future perspectives. Future Microbiol 2014; 9: 683695. 
38. De Pauw B, Walsh TJ, Donnelly JP, et al; European Organization for Research and Treatment of Cancer/Invasive Fungal Infections Cooperative Group; National Institute of Allergy and Infectious Diseases Mycoses Study Group (EORTC/MSG) Consensus Group. Revised definitions of invasine fungal disease from the European Organization for Research and Treatment of Cancer/Invasive Fungal Infections Cooperative Group and the National Institute of Allergy and Infectious Diseases Mycoses Study Group (EORTC/MSG) Consensus Group. Clin Infect Dis 2008; 46: 1813-1821.

39. Iosifidis E, Papachristou S, Roilides E. Advances in the treatment of mycoses in pediatric patients. J Fungi (Basel) 2018; 4: 115.

40. Vehreschild JJ, Birtel A, Vehreschild MJ, et al. Mucormycosis treated with posaconazole: review of 96 case reports. Crit Rev Microbiol 2013; 39: 310-324.

41. Pagano L, Cornely OA, Busca A, et al. Combined antifungal approach for the treatment of invasive mucormycosis in patients with hematologic diseases: a report from the SEIFEM and FUNGISCOPE registries. Haematologica 2013; 98: e127-e130.
42. Kyvernitakis A, Torres HA, Jiang Y, Chamilos G, Lewis RE, Kontoyiannis DP. Initial use of combination treatment does not impact survival of 106 patients with haematologic malignancies and mucormycosis: a propensity score analysis. Clin Microbiol Infect 2016; 22: 811.e1-811.e8.

43. Rai S, Yadav S, Kumar D, Kumar V, Rattan V.. Management of rhinomaxillary mucormycosis with posaconazole in immunocompetent patients. J Oral Biol Craniofac Res 2016; 6(Supp 1): S5-S8.

44. Spellberg B, Kontoyiannis DP, Fredricks D, et al. Risk factors for mortality in patients with mucormycosis. Med Mycol 2012; 50: 611-618.

45. Madney Y, Khedr R, Ahmed N, et al. Overview and outcome of mucormycosis among children with cancer: report from the Children's Cancer Hospital Egypt. Mycoses 2019; 62: 984-989.

46. Sipsas NV, Gamaletsou MN, Anastasopoulou A, Kontoyiannis DP.Therapy of mucormycosis. J Fungi (Basel) 2018; 4: 90. 